\title{
Semen analysis of Golden Retriever healthy dogs and those affected by muscular dystrophy
}

\author{
M. A. Peres ${ }^{1,2}$, A. M. da Rocha ${ }^{3}$, C. I. Vannucchi ${ }^{2}$, C. M. Mendes ${ }^{2}$, P. V. Cavalcanti ${ }^{2}$, M. Nichi², \\ C. E. Ambrosio ${ }^{4}$, M. A. Miglino ${ }^{1}$, J. A. Visintin ${ }^{2}$ \& M. E. O. D'Ávila Assumpção ${ }^{2}$ \\ 1 Department of Surgery, School of Veterinary Medicine and Animal Science, University of São Paulo, São Paulo, Brazil; \\ 2 Department of Animal Reproduction, School of Veterinary Medicine and Animal Science, University of São Paulo, São Paulo, Brazil; \\ 3 Department of Cell and Developmental Biology, Medical School, University of Michigan, Ann Arbor, MI, USA; \\ 4 Department of Basic Sciences, School of Animal Science and Food Engineering, University of São Paulo, São Paulo, Brazil
}

\author{
Keywords \\ Dog semen-flow cytometry-GRMD— \\ ROS-TBARS

\section{Correspondence \\ Dr Maria Angélica Peres, Department of \\ Surgery, School of Veterinary Medicine and \\ Animal Science, São Paulo University, Av Prof \\ Dr Orlando Marques de Paiva, 87 São Paulo, \\ São Paulo 05508-270, Brazil. \\ Tel.: +55-11-30917665; \\ Fax: +55-11-30917412; \\ E-mail: map@usp.br}

Accepted: December 28, 2012

doi: 10.1111/and.12079

\begin{abstract}
Summary
This study aimed to characterise canine flow cytometry semen analysis, as well as seminal reactive oxygen species dosage using the Golden Retriever breed as model of study. Moreover, we searched for the influence of muscular dystrophy in Golden Retriever dogs on semen parameters. Thirty-seven semen samples were obtained from healthy Golden Retrievers $(n=15)$ and from muscular dystrophy affected dogs $(n=22)$. Sperm-rich fractions were analysed by standardised breeding soundness examination in addition to the assay of fluorescence assisted cell sorting for acrosome integrity, mitochondrial activity and DNA fragmentation. Volume of ejaculate, per cent of motile spermatozoa and vigour were similar between groups; there were no differences in the per cent of minor and major defects. Integrity of acrosomal membrane, mitochondrial potential and sperm DNA fragmentation had no significant differences between groups either. Animals from control group had higher concentration of spontaneous seminal oxidative species in comparison with affected animals. Dogs affected by dystrophy had seminal parameters similar to those observed in healthy dogs except for the lower concentration of oxidative species. Future studies aiming to establish reference values for canine seminal parameters should be considered preferably with distinction of breeds.
\end{abstract}

\section{Introduction}

Precise methods that can standardise all the parameters of semen analysis have been used for the last decades in many animal species. These methods can eliminate the variations of individual interpretation and can bring more certainty to results. In regard to the canine species, semen should be analysed by methods that could establish defined parameters once there is evidence that breed particularities can exist and can affect the interpretation of results (Peña et al., 2001; Rijsselaere et al., 2005; England et al., 2010).

Flow cytometry has the advantage of been highly quantitative, repeatable and a sensitive technique capable of characterising a large number of spermatozoa in a short period of time. With the use of different fluorescent markers, sperm structures and functions can be analysed in a detailed manner (Peña et al., 2001; Rijsselaere et al., 2005; Cheuquemán et al., 2012). For example, mitochon- drial membrane potential can be assessed to demonstrate its activity, as the higher the potential the greater the energy produced to promote sperm motility. In addition, the assessment of acrosome and sperm DNA integrity can be very important to consider the capacity of fertilisation of a semen sample and determinate the reproductive capacity of an individual (Cheuquemán et al., 2012; Minervini et al., 2013).

Another important parameter to be evaluated is the production of reactive oxygen species (ROS), which are active molecules produced during the oxygen reduction. Sperm cells are particularly susceptible to peroxidative damage once they have high content of polyunsaturated fatty acids in their membrane, and these molecules are the target to free radicals generated by peroxidation. Low and controlled concentration of ROS play an important role in sperm physiology but, when produced at high concentrations, ROS can be implicated in several deleterious effects on the function and 
viability of spermatozoa (Cassani et al., 2005; Nichi et al., 2007).

Golden Retriever is a common and widespread breed. However, they can present an inherited muscular dystrophy called Golden Retriever's Muscular Dystrophy (GRMD), which is a degenerative myopathie caused by mutations in the dystrophin gene. Dystrophin is a cytoskeletal protein that provides the link between internal cytoskeleton of the muscular cell and the extracellular matrix and its absence is responsible for Duchenne's and Becker's Muscular Dystrophy in humans (DMD and BMD respectively) (Collins \& Morgan, 2003; Vainzof \& Zatz, 2003; Deconick \& Dan, 2007). Short dystrophin products are expressed in a variety of organs such as central nervous system, kidney, liver, lung, thymus, intestine, eyes, testis and the sperm membrane (Torkatz et al., 1998; Hernández-González et al., 2005). These shorter isoforms may have special roles in specific manifestations in patients affected by DMD and BMD (Torkatz et al., 1998).

Routine evaluation by spermiogram can be insufficient to detect the effects of dystrophy in sperm parameters. Hence, precise techniques such as flow cytometry can be valuable to understand the mechanisms of sperm development and contribute to the overall knowledge of the disease. Muscular cells from dystrophic patients are known to be more sensitive to oxidative effects once their membrane structure is altered by the absence of dystrophin, so alterations in ROS concentration could be important in this model (Reggiani, 2008; Whitehead et al., 2008). GRMD dogs are the closest animal model to human DMD and BMD. They are able to naturally reproduce and are suitable for the study and characterisation of semen (Collins \& Morgan, 2003).

The aim of this study was to characterise Golden Retriever semen and the influence of muscular dystrophy in semen attributes measured by flow cytometry and ROS generation.

\section{Materials and methods}

\section{Ethics}

This study was approved by the Review Board of the School of Veterinary Medicine and Animal Science of the University of Sao Paulo, Brazil (Process N 1011/2006).

\section{Materials}

All the chemicals used in this study were purchased from Sigma-Aldrich Corp. (St Louis, MO, USA), unless otherwise specified.

\section{Animals}

Four healthy Golden Retrievers [control group (CG)] and five GRMD dogs [affected group (AF) 1-5] ageing from 1.5 to 4.5 years were used in this study. Affected dogs were housed at 'Brazil GRMD Kennel' located at the School of Veterinary Medicine and Animal Science of the University of São Paulo, Brazil. All dogs comprised in the CG group were privately owned and clinically healthy. The diagnosis of muscular dystrophy was performed as previously reported by Zucconi et al. (2011).

Dogs affected by GRMD were phenotypically classified as mild (grade I), moderate (grade II) or severe (grade III). Classification was according to muscular and skeletal symmetry, gastrointestinal alterations, cardiac problems and dehydration. Two dogs presented Grade I physical manifestation (AF 2 and AF 5), one was Grade II (AF 4) and two presented Grade III manifestation (AF 1 and AF 3) (Ambrosio et al., 2009).

Fertility was proved in two animals of the AF group (AF1 - one litter with seven puppies after artificial insemination; AF2 - five litters by natural copulation). One animal from CG procreated prior to this work and had a litter of seven healthy puppies. Another dog from CG bred after the end of this research and had six healthy pappies. The other animals did not have the opportunity to copulate until this report.

\section{Semen collection}

Semen samples were obtained by digital manipulation, monthly, in five replicates. Once dog ejaculate presents three fractions that can be recognised by its colour and been the second fraction the one that contains spermatozoa, the sperm-rich fraction, this second fraction was the only portion of ejaculate collected in plastic tubes via glass funnel.

\section{Sperm analysis}

All samples were processed and analysed by the same theriogenologist. Initial analysis of the sperm-rich fraction comprised volume determination, per cent of straightforward motility $(0-100 \%)$ and vigour $(0-5)$. These evaluations were performed under light microscope $(\times 200$ magnification), at $37^{\circ} \mathrm{C}$. Sperm motility is expressed as the percentage of total progressively motile spermatozoa and was analysed in a drop of semen put between a slide and a coverslip. Vigour is referred to the force of head movement of the sperm been classified as ' 0 - no movement' to '5 - very vigorous'.

Sperm morphology was assessed in samples fixed in $10 \%$ phormol-saline solution (PS) under phase contrast 
microscopy and through eosin-nigrosin (EN) stained smears under light microscopy ( $\times 1000$ magnification). Smears stained by EN were also used to evaluate the per cent of dead/alive cells (Björndhal et al., 2003). Sperm concentration was calculated in Neubauer chamber, and sperm defects were classified as major, when related to spermatogenesis and minor, when related to epididymis transport and storage (Vannucchi et al., 1998).

Fluorescence assisted cell sorting (FACS) was performed with FACSCalibur flow cytometer (Becton Dickinson, Franklin Lakes, NJ, USA) configured according to previous experiments (Peña et al., 1999; Evenson et al., 2002; Martinez-Pastor et al., 2004; Celeghini et al., 2007). Analyses were applied in $10^{4}$ spermatozoa, from a gate which contained only sperm cells, pre-defined previously by cell sorting analysis.

\section{Evaluation of acrosome integrity and mitochondrial} activity

After centrifugation (300 $g$ for $5 \mathrm{~min}$ ), semen samples were resuspended in phosphate-buffered saline (PBS) to the final concentration of $7 \times 10^{6}$ cells ml ${ }^{-1}$. Fluorescein isothiocyanate (FITC)-conjugated Pisum sativum agglutinin (PSA) was used to evaluate acrosome integrity. Cells with disrupted acrosome (reacted or reacting acrosome) bind to PSA emitting green fluorescence whereas intact acrosome does not show fluorescence. Fifty microlitres $(50 \mu \mathrm{l})$ of FITC-PSA $\left(100 \mu \mathrm{g} \mathrm{ml}^{-1}\right)$ was mixed with $150 \mu \mathrm{l}$ of semen, containing approximately $10^{6}$ spermatozoa. The mixture was incubated at $37^{\circ} \mathrm{C}$, in dark room for $10 \mathrm{~min}$.

JC-1 is a fluorescent probe used to verify mitochondrial function which separates two groups of cells by colour code. JC-1 accumulates inside the cells with high mitochondrial membrane potential, forming aggregates that dye in orange/red, and in cells with low mitochondrial membrane potential, JC-1 probe forms monomers and dye in green (Martinez-Pastor et al., 2004; Celeghini et al., 2007; Minervini et al., 2013). A second $150 \mu \mathrm{l}$ aliquot of semen was incubated, for $10 \mathrm{~min}$, with $2 \mu \mathrm{l}$ of JC-1 (5,5',6,6'-tetrachloro-1,1',3,3'tetraethylbenzimidazolyl-carbocyanine iodide - 5\%) (Molecular Probes, Invitrogen, Carlsbad, CA, USA).

The FACS analysis for acrosome integrity (FITC-PSA) and mitochondrial activity (JC-1) were evaluated on FCS EXPRESS software (DENOVO Software, Thornhill, ON, Canada).

\section{Sperm chromatin structure evaluation}

The status of chromatin condensation was checked following the procedure described by Evenson et al. (2002).
Semen sample was prepared with $5 \times 10^{6}$ cells ml $^{-1}$ resuspended in TNE buffer $(0.01 \mathrm{M}$ Tris- $\mathrm{HCl}, 0.015 \mathrm{M}$ $\mathrm{NaCl}$ and $1 \mathrm{~mm}$ disodium EDTA, pH 7.4). An aliquot of $200 \mu \mathrm{l}$ of the diluted sample was mixed with $400 \mu \mathrm{l}$ of acid detergent buffer $(0.08 \mathrm{~N} \mathrm{HCl}, 0.15 \mathrm{~m} \mathrm{NaCl}, 0.1 \%$ Triton X 100, pH 1.2). Thirty seconds later, $1.20 \mathrm{ml}$ of acridine orange $(\mathrm{AO})$ staining solution $(0.037 \mathrm{M}$ citric acid, $0.126 \mathrm{M} \mathrm{Na} 2 \mathrm{HPO}_{4}, 0.0011 \mathrm{M}$ disodium EDTA, $0.15 \mathrm{M} \mathrm{NaCl}, \mathrm{pH} 6.0)$ containing $6 \mu \mathrm{g} \mathrm{ml}^{-1}$ of $\mathrm{AO}$ was added. This mixture was incubated for 3-5 min and analysed by flow cytometry. When AO penetrates the nucleus of the sperm cell and find a condensed, double-stranded DNA, it will emit a green fluorescence. When the DNA is damaged, in single-stranded form, the AO molecules will agglutinate and emit red fluorescence. DNA fragmentation index (DFI) was calculated based on the percentage of spermatozoa outside the main population in a histogram of $\alpha \mathrm{T}$ (ratio between red fluorescence and total fluorescence) (Evenson \& Jost, 2000; Minervini et al., 2013). DNA fragmentation was then calculated with FLowJo Version Mac (Flow Cytometry Analysis Software; Tree Star Inc., Ashland, OR, USA).

\section{Oxidative stress}

For the evaluation of the sperm resistance to oxidative stress (OS), the procedure was conducted following the protocol described by Nichi et al. (2007). Following semen centrifugation, the supernatant (seminal plasma) was frozen, immediately after collection to analyse the spontaneous lipid peroxidation [spontaneous thiobarbituric acid reactive substances (TBARS)]. For the analysis of the susceptibility of the sperm sample against the OS (induced TBARS), semen was resuspended in PBS to a final concentration of $7 \times 10^{6}$ cells $\mathrm{ml}^{-1}$. Lipid peroxidation was then induced by adding ferrous sulphate $(250 \mu \mathrm{l}, 4 \mathrm{~mm})$ and sodium ascorbate $(250 \mu \mathrm{l}, 20 \mathrm{~mm})$ to $1 \mathrm{ml}$ of the sperm suspension. The mixture was incubated for $1.5 \mathrm{~h}$ at $37^{\circ} \mathrm{C}$. After the incubation period, for the induced TBARS, and after seminal plasma thawing, for the spontaneous TBARS, proteins were precipitated by adding trichloroacetic acid (TCA 10\%) in a $2: 1$ proportion on both, incubation mixture and seminal plasma. Both mixtures were centrifuged (18 $000 \mathrm{~g}, 15 \mathrm{~min}$ ), and $500 \mu$ of the supernatant was mixed with $500 \mu$ l of $1 \%$ (v:v) thiobarbituric acid (TBA, 1\% diluted in $0.05 \mathrm{~N}$ $\mathrm{NaOH})$ in a glass tube and placed into a boiling water bath $\left(100{ }^{\circ} \mathrm{C}\right)$ for $20 \mathrm{~min}$. The solution was immediately cooled in an ice bath $\left(0^{\circ} \mathrm{C}\right)$ to stop chemical reaction. The TBARS were then quantified using a spectrophotometer (U-2001 spectrophotometer; Hitachi High Technologies America, Inc., San Jose, CA, USA), at a $532 \mathrm{~nm}$ wavelength. 


\section{Statistical analysis}

Data were evaluated using SAS System for Windows (SAS Institute Inc., Cary, NC, USA). No effect of repetition or interaction between group and repetitions was observed for all variables. Therefore, the main effect of 'Group' was evaluated and considered. Parametric (Student's $t$-test) and nonparametric (Mann-Whitney $U$-test) tests were applied, according to the residue normality (Gaussian distribution) and variance homogeneity of each variable. A probability value of $P<0.05$ was considered statistically significant. Results are reported as untransformed means \pm SEM.

\section{Results}

Animals comprised in control and AFs were easily conditioned to the semen collection procedure and presented normal libido. However, animal AF3 experienced difficulty in maintaining penis erection and ejaculation during one episode of semen collection.

Thirty-seven semen samples were obtained during this study ( $\mathrm{CG}=15$ ejaculates and $\mathrm{AF}=22$ ejaculates). The five ejaculates that are missed in CG are due to problems in the availability of the animals on the day of the procedure (two animals missed on day 1; one animal on day 2; one on day 4; and one on day 5). In the AF group, the loss of three collections occurred once due to difficulty in ejaculation, as described before, and twice the animals
AF5 was considered not in good health for the procedure, once this animal was not feeding properly in the 2 or 3 days prior to the date of semen collection, as a consequence of the dystrophy. Sperm analysis comprising sperm concentration, motility, vigour and morphology was performed for all ejaculates. Volume of ejaculate, per cent of motile spermatozoa and vigour were similar between groups; furthermore, there were no differences in the per cent of minor and major defects observed in preparations with PS and EN (Table 1).

Fluorescence assisted cell sorting was performed for assessment of integrity of acrosomal membrane, mitochondrial potential and sperm DNA fragmentation, but no significant differences between groups were observed (Table 2).

Regarding the OS evaluation, animals from CG had higher concentration of spontaneous seminal TBARS in comparison with affected animals $(P=0.006)$ (Table 2).

\section{Discussion}

This study evaluated seminal parameters from four healthy Golden Retriever dogs and five GRMD affected dogs.

Seminal analysis indicated that the volume of the ejaculate, sperm concentration, motility, vigour and morphology obtained was within the normal range for the species (Vannucchi et al., 1998), except for dog AF1 which had

Table 1 Descriptive analysis of routine sperm evaluation (mean \pm SEM)

\begin{tabular}{|c|c|c|c|c|c|c|c|c|c|c|}
\hline \multirow[b]{3}{*}{ Group } & \multirow[b]{3}{*}{$\mathrm{N}$} & \multirow{3}{*}{$\begin{array}{l}\text { Volume } \\
\text { (ml) }\end{array}$} & \multirow{3}{*}{$\begin{array}{l}\text { Motility } \\
(\%)\end{array}$} & \multirow{3}{*}{$\begin{array}{l}\text { Vigour } \\
(1-5)\end{array}$} & \multirow{3}{*}{$\begin{array}{l}\text { Concentration } \\
\left(\times 10^{6} \text { spermatozoa }\right. \\
\text { per Ejaculate })\end{array}$} & \multirow{3}{*}{$\begin{array}{l}\text { Alive } \\
(\%)\end{array}$} & \multicolumn{4}{|l|}{ Defects } \\
\hline & & & & & & & \multicolumn{2}{|l|}{ PS(\%) } & \multicolumn{2}{|l|}{$\mathrm{EN}(\%)$} \\
\hline & & & & & & & Major & Minor & Major & Minor \\
\hline $\mathrm{AF}$ & 5 & $1.2 \pm 0.1$ & $83.9 \pm 1.4$ & $3.6 \pm 0.1$ & $661.8 \pm 87.7$ & $95.6 \pm 0.8$ & $6.3 \pm 1.5$ & $5.7 \pm 1.4$ & $12.8 \pm 1.5$ & $1.1 \pm 0.3$ \\
\hline CG & 4 & $1.0 \pm 0.1$ & $85.4 \pm 1.8$ & $3.6 \pm 0.2$ & $410.4 \pm 70.6$ & $93.1 \pm 2.6$ & $3.8 \pm 0.9$ & $7.1 \pm 1.7$ & $8.5 \pm 1.0$ & $1.5 \pm 0.4$ \\
\hline
\end{tabular}

PS, phormol saline; EN, Eosin-nigrosin; AF, affected group; CG, control group.

a,b Different superscripts within a column indicate significant differences $(P<0.05)$ between groups.

Table 2 Fluorescence assisted cell sorting analysis showing the percentage of spermatozoa with damaged acrosome membrane (FITC-PSA), high mitochondrial potential (JC-1), fragmented DNA and levels of spontaneous and induced thiobarbituric acid reactive substances (TBARS) $($ mean \pm SEM)

\begin{tabular}{llllll}
\hline & $\begin{array}{l}\text { FITC-PSA } \\
\text { (\% damaged }\end{array}$ & $\begin{array}{l}\text { JC1 } \\
\text { (\% high mitochondrial } \\
\text { potential) }\end{array}$ & $\begin{array}{l}\text { DNA fragmentation } \\
(\%)\end{array}$ & $\begin{array}{l}\text { Spontaneous TBARS } \\
\left(\mathrm{ng} \mathrm{m}^{-1}\right)\end{array}$ & $\begin{array}{l}\text { Induced TBARS } \\
\text { (ng 10 }\end{array}$ \\
\hline AF & $25.0 \pm 2.2$ & $68.7 \pm 1.2$ & $4.6 \pm 0.7$ & $188.9 \pm 28.1^{\mathrm{a}}$ & $600.0 \pm 40.2$ \\
CG & $32.5 \pm 3.9$ & $60.5 \pm 4.5$ & $6.5 \pm 1.0$ & $287.5 \pm 39.7^{\mathrm{b}}$ & $655.7 \pm 93.7$ \\
\hline
\end{tabular}

$A F$, affected group; $C G$, control group.

${ }^{\mathrm{a}, \mathrm{b}}$ Different superscripts within a column indicate significant differences $(P<0.05)$ between group. 
teratozoospermia (more than 20\% of spermatozoa total defects) due to major defects (e.g. pear shaped and giant sperm heads). The AF1 dog showed a slightly small volume of semen, and we speculated the possibility of involvement of an inefficient contraction of the prostate muscles. These muscles are very important on the ejaculation process once they secrete prostatic fluid into the urethra and may also help expel the ejaculate. This characteristic of the affected dogs can be described as one of the symptoms of the muscular disease and can be responsible for a higher sperm concentration, otherwise nonsignificant, in the AF group when compared with CG group.

The constant stress that GRMD dogs are subjected might be responsible for the high incidence of sperm defects. In dogs, as well as other mammals including men, inadequate thermoregulation of the testis can lead to impaired spermatogenesis and the increase in major sperm defects (Barth \& Oko, 1989; Hansen, 2009). Luppi et al. (2007) observed histological alterations on the cremaster muscle from GRMD dogs. This muscle is partially responsible for the thermoregulation of the testis, and the loss of tonus in affected dogs might lead to a slight decrease in the testis temperature, though, in this group of animal without imprinting significant effects on spermatozoa's morphology, acrosomal integrity and mitochondrial activity. In São Paulo, Brazil, where these animals were placed, the annual mean temperature is between $17-23^{\circ} \mathrm{C}$; so, the more distant position of the testis in relation to the body of the animal has minimal interference in sperm quality but it is possible that in colder areas this effect could be detected.

During the past decade, flow cytometry have demonstrated to be an excellent tool to semen evaluation. However, we observed that in spite of a teratozoospermic dog in the AF group, no differences in seminal characteristics evaluated by FACS were verified. The high percentage of pear shaped spermatozoa and giant head spermatozoa detected on animal AF1 did not correlate to acrosome integrity or DNA fragmentation.

Although few researches have been performed with flow cytometry and dog semen, the acrosome integrity findings in our study (means $67.5 \%$ and $75 \%$, respectively, CG for and AF groups) are in accordance with other experiments (Peña et al., 1999; Cheuquemán et al., 2012).

For DNA fragmentation, according to references range for human semen, the fragmentation is considered low, thus ultimately not affecting the fertility of both groups (Evenson \& Jost, 2000).

Neagu et al. (2011) demonstrated that among dogs, there are differences in the basal levels of lipid peroxidation. Potentially, dystrophin alterations can also alter the tensions and fluidity on sperm membrane, as described for Myotonic Muscular Dystrophy human patients (Hortas et al., 2000). Based on such statement, we can infer that the decreased ROS concentration in GRMD affected dogs compared with CG group might be due to a lower sperm activity or changes in the plasma membrane composition. Thus, the lower proportions of polyunsaturated fatty acids in GRMD spermatozoa can be responsible for distinct membrane fluidity and, ultimately, a lower source of ROS production.

It is well known that the membrane fluidity is essential for spermatozoa to develop all stages until egg fertilisation, such as capacitation, acrosome reaction and egg binding. Therefore, it must be emphasised that experiments on sperm capacitation and fertilising ability in GRMD dogs should be conducted to clarify this important issue of sperm physiology (Maneesh \& Jayalekshmi, 2006; Nichi et al., 2007; Neagu et al., 2011).

Hernández-González et al. (2005) demonstrated the absence of a short dystrophin product encoded by an internal promoter of DMD gene, located in the sperm flagellum in $\mathrm{mdx}^{3 \mathrm{cv}}$ mouse. The authors related the absence of Dp71 to the increase in the proportion of spermatozoa with alterations in the flagellar morphology, as well as sperm capacitation, acrosome reaction and motility. On the other hand, this type of morphological and functional sperm defect was not observed in the present study with GRMD dogs, as acrosome reaction and sperm motility were not different between groups. Our findings suggest that Dp71 might have distinct roles among species, and studies addressing the presence and function of Dp71 in dogs should be undertaken.

In conclusion, dogs affected by GRMD have seminal parameters similar to those observed in Golden Retriever healthy dogs, except for the lower concentration of TBARS. These findings can be considered subtle; however, the decreased oxidative activity might be related to impaired acrosomal reaction and sperm capacitation. We therefore suggest that future studies aiming to establish reference values for canine seminal parameters should be considered preferably with the distinction of breeds.

\section{Acknowledgements}

We thank Prof. Luiz Carlos de Sá Rocha for the use of flow cytometer and Prof. Valquiria Hyppolito Barnabé for the ROS analysis.

This study was partially supported by FAPESP, 2006/ 50272-3.

\section{References}

Ambrosio CE, Fadel L, Gaiad TP, Martins DS, Araújo KPC, Zucconi E, Brolio MP, Giglio RF, Morini AC, Jazedje T, 
Froes TR, Feitosa MLT, Valadares MC, Beltrão-Braga PCB, Meirelles FV, Miglino MA (2009) Identification of three distinguishable phenotypes in golden retriever muscular dystrophy. Genet Mol Res 8:389-396.

Barth AD, Oko RJ (1989) Defects of the sperm head. In: Abnormal morphology of bovine spermatozoa. Barth AD, Oko RJ (eds). IS Iowa State University Press, Ames, pp. 130-192.

Björndhal L, Söderlund I, Kvist U (2003) Evaluation of the one-step eosin-nigrosin staining technique for human sperm vitality assessment. Hum Reprod 18:813-816.

Cassani P, Beconi MT, O'Flaherty C (2005) Relationship between total superoxide dismutase activity with lipid peroxidation, dynamics and morphological parameters in canine semen. Anim Reprod Sci 86:163-173.

Celeghini ECC, de Arruda RP, de Andrade AFC, Nascimento J, Raphael CF (2007) Practical techniques for bovine sperm simultaneous fluorimetric assessment of plasma, acrosomal and mitochondrial membranes. Reprod Domest Anim 42:479-488.

Cheuquemán C, Bravo P, Treulén F, Giojalas L, Villegas J, Sánchez R, Risopatrón J (2012) Sperm membrane functionality in the dog assessed by flow cytometry. Reprod Domest Anim 47:39-43.

Collins CA, Morgan JE (2003) Duchenne's muscular dystrophy: animal models used to investigate pathogenesis and develop therapeutic strategies. Int J Exp Pathol 84:165-172.

Deconick N, Dan B (2007) Pathophysiology of duchenne muscular dystrophy: current hypotheses. Pediatr Neurol 36: $1-7$.

England GC, Phillips L, Freeman SL (2010) Heritability of semen characteristics in dogs. Theriogenology 74:1136-1140.

Evenson D, Jost L (2000) Sperm chromatin structure assay is useful for fertility assessment. Methods Cell Sci 22:169-189.

Evenson DP, Larson KL, Jost LK (2002) Sperm Chromatin Structure Assay: its clinical use for detecting sperm DNA fragmentation in male infertility and comparison with other technique. J Androl 23:25-43.

Hansen PJ (2009) Effects of heat stress on mammalian reproduction. Philos Trans R Soc Lond B Biol Sci 364: 3341-3350.

Hernández-González EO, Mornet D, Rendon A, MartínezRojas D (2005) Absence of Dp71 in $\mathrm{mdx}^{3 \mathrm{cv}}$ mouse spermatozoa alters flagelar morphology and the distribution of ion channels and nNOS. J Cell Sci 118:137-145.

Hortas ML, Castilla JA, Gil MT, Molina J, Garrido ML, Morell M, Redondo M (2000) Decreased sperm function of patients with myotonic muscular dystrophy. Hum Reprod 15:445-448.

Luppi MMCP, Vannucchi CI, Ambrosio CE, Morini AC, Pessuto EC, Lourenço TPM, Miglino MA(2007) Avaliação andrológica de cães da raça golden retriever sadios e afetados pela distrofia muscular. Anais do $7^{\circ}$ Congresso Paulista de Clínicos de Pequenos Animais, São Paulo.

Maneesh M, Jayalekshmi H (2006) Role of reactive oxygen species and antioxidants on pathophysiology of male reproduction. Indian J Clin Biochem 21:80-89.
Martinez-Pastor F, Johannisson A, Gil J, Kaabi M, Anel L, Paz P, Rodriguez-Martinez H (2004) Use of chromatin stability assay, mitochondrial stain JC-1, and fluorometric assessment of plasma membrane to evaluate frozen-thawed RAM semen. Anim Reprod Sci 84:121-133.

Minervini F, Guastamacchia R, Pizzi F, Dell'aquila M, Barile V (2013) Assessment of different functional parameters of frozen-thawed buffalo spermatozoa by using cytofluorimetric determinations. Reprod Domest Anim 48:317-324.

Neagu VR, Macías Garcíaa B, Morillo Rodrígueza A, Ortega Ferrusolaa C, Gallardo Bolañosa JM, González Fernándezb L, Tapiab JA, Peñaa FJ (2011) Determination of glutation peroxidase and superoxide dismutase activities in canine seminal plasma and its relation with sperm quality and lipid peroxidation post thaw.

Theriogenology 75:10-16.

Nichi M, Goovaerts IGF, Cortada CNM, Barnabe VH, De Clercq JBP, Bols PEJ (2007) Roles of lipid peroxidation and cytoplasmic droplets on in vitro fertilization capacity of sperm colleted from bovine epididymides stored at 4 and $34{ }^{\circ} \mathrm{C}$. Theriogenology 67:334-340.

Peña A, Johanninsson A, Linde-Forsberg C (1999) Postthaw evaluation of dog spermatozoa using new triple fluorescent staining and flow cytometry. Theriogenology 52:965-980.

Peña AI, Johannisson A, Linde-Forsberg C (2001) Validation of flow cytometry for assessment of viability and acrosomal integrity of dog spermatozoa and for evaluation of different methods of cryopreservation. J Reprod Fertil Suppl 57: 371-376.

Reggiani C (2008) Between channels and tears: aim at ROS to save the membrane of dystrophic fibres. J Physiol 586:1779.

Rijsselaere T, van Soom A, Tanghe S, Soryn M, Maes D, de Kruif A (2005) New techniques for the assessment of canine semen quality: a review. Theriogenology 64:706-719.

Torkatz SA, Dincan NM, Rash SM, Sadeghi A, Dewan AK, Pillers DAM (1998) Redefinition of dystrophin isoform distribution in mouse tissue by RT-PCR implies role in nonmuscle manifestations of Duchenne muscular dystrophy. Mol Genet Metab 65:272-281.

Vainzof M, Zatz M (2003) Protein defects in neuromuscular diseases. Braz J Med Biol Res 36:543-555.

Vannucchi CI, Satzinger S, Santos SEC (1998) Avaliação seminal em cães - aspectos práticos. Clin Vet 15:22-26.

Whitehead NP, Pham C, Gervasio OL, Allen DG (2008) $\mathrm{N}$-Acetylcysteine ameliorates skeletal muscle pathophysiology in mdx mice. J Physiol 586:2003-2014.

Zucconi E, Vieira NM, Bueno Jr CB, Secco M, Jazedje T, Valadares MC, Suzuki MF, Bartolini P, Vainzof M, Zatz M (2011) Preclinical studies with umbilical cord mesenchymal stromal cells in different animal models for muscular dystrophy. J Biomed Biotechnol 2011:9. 